\title{
2 Automatisiertes und vernetztes Fahren: Berücksichtigung des lokalen, räumlichen Kontextes und räumliche Differenzierung
}

Emilia M. Bruck, Aggelos Soteropoulos

$\begin{array}{lll}\text { 1. } & \text { Einleitung } & 8\end{array}$

2. Räumliche Differenzierung und Raumtypen 9

3. Räumliche Wirkungsweisen des automatisierten Fahrens 10

3.1 Primäre räumliche Wirkungen 12

3.2 Sekundäre räumliche Wirkungen 12

4. Räumliche differenzierte Wirkungsweisen des automatisierten Fahrens 14

$\begin{array}{ll}\text { Literatur } & 15\end{array}$

Emilia M. Bruck

TU Wien, future.lab Research Center und Forschungsbereich Örtliche Raumplanung (IFOER) emilia.bruck@tuwien.ac.at

Aggelos Soteropoulos

TU Wien, future.lab Research Center und Forschungsbereich Verkehrssystemplanung (IVS)

aggelos.soteropoulos@tuwien.ac.at

(C) Der/die Autor(en) 2021

M. Mitteregger et al. (Hrsg.), AVENUE21. Politische und

planerische Aspekte der automatisierten Mobilität,

https://doi.org/10.1007/978-3-662-63354-0_2 


\section{EINLEITUNG}

Die Beiträge der vorliegenden Anthologie behandeln Raumwirkungen sowie Steuerungs- und Planungsansätze auf kommunaler und regionaler Ebene. Hierbei erweist es sich als unumgänglich, die räumlichen Voraussetzungen ländlich geprägter Gemeinden und Regionen von jenen der urbanen Zentren und Großstädte zu unterscheiden. Ländliche sowie urbane Räume sind nicht homogen, sondern vielschichtige Gebiete mit unterschiedlichen siedlungsstrukturellen, naturräumlichen und infrastrukturellen Ausstattungen, Funktionen und Herausforderungen (VCÖ 2019: 9). Ihre Charakteristika schaffen unterschiedliche Ausgangslagen für den Einsatz des automatisierten und vernetzten Fahrens und wirken in Folge auf dessen räumliche Effekte, die sich in einzelnen Gebieten lokal spezifisch ausprägen werden.

Räumliche Strukturen und Mobilitätsverhalten (Modal Split, Motorisierungsgrad, $\mathrm{CO}_{2}$-Emissionen) sowie wirtschaftliche und infrastrukturelle Gegebenheiten sind nur einige der Faktoren, welche die Voraussetzungen für den Einsatz automatisierter Anwendungsformen bestimmen. Ob der Differenzen ist zu berücksichtigen, dass Handlungsansätze für die Mobilitäts- und Siedlungsentwicklung auch in Hinblick auf das automatisierte Fahren nur bedingt von urbanen Zentren auf Regionen und ländliche Räume übertragbar sein werden. Eine räumlich differenzierte Betrachtung kann dazu beitragen, mögliche Wirkungsweisen vor dem Hintergrund lokaler Gegebenheiten zu prüfen und planerisches Handeln entsprechend anzupassen.

Voraussetzung hierfür ist die Anerkennung der raumbildprägenden wechselseitigen Wirkungsbeziehung zwischen Verkehrsmittel und Siedlungsentwicklung sowie Straßenräumen und Stadtgestalt (Angerer/Hadler 2005; Mitteregger et al. 2020: 67). So wie die Einführung der Eisenbahn oder des Automobils zu einem historischen Wandel der Erreichbarkeiten und der Siedlungsentwicklung geführt hat, so birgt auch das automatisierte und vernetzte Fahren das Potential, öffentliche Räume, Orts- und Landschaftsbilder sowie Siedlungsstrukturen grundlegend zu verändern. Umgekehrt kann eine Veränderung in der Raumnutzung bzw. der Siedlungsstruktur zu einer Änderung der Erreichbarkeitsverhältnisse und in Folge auch zu einer relativ schnellen Adaptierung der Mobilitätsaktivitäten führen (Wegener/Fürst 1999; Bertolini 2012, 2017). Inwieweit sich die Wirkungen neuer Verkehrsmittel und Verkehrsinfrastrukturen räumlich manifestieren, wird je nach siedlungsstrukturellem Kontext variieren: So besteht beispielsweise ein Zusammenhang zwischen der Wirksamkeit von Verkehrsinfrastrukturinvestitionen, dem Entwicklungsgrad des Siedlungsgebiets und dessen aktueller Erreichbarkeit.

Während in Gebieten hoher Erreichbarkeit und/oder bereits stark entwickelter Siedlungsstruktur Sättigungseffekte bei der Verbesserung der Erreichbarkeit auftreten, sind in wenig entwickelten Gebieten stärkere Effekte durch neue Verkehrsinfrastrukturen oder neue Verkehrsmittel zu erwarten, da die Erreichbarkeit erkennbar gehoben werden kann (Kasraian et al. 2016, Mitteregger et al. 2020). Für ein differenziertes Verständnis von räumlichen Veränderungen in Wechselwirkung mit neuen Mobilitätsformen sind Siedlungs- und Infrastrukturräume sowohl in ihren funktionalen und strukturellen Unterschieden als auch in ihrem historischen Kontext, den jeweiligen Entstehungsbedingungen, spezifisch wahrzunehmen, um auf Defizite und Potentiale gezielt eingehen zu können. Letztlich gilt es auch, die Abhängigkeit zwischen räumlichen Entwicklungsprozessen und Planungs-, Mobilitäts- sowie Siedlungspolitik zu nennen - siehe dazu Teil 4 „Governance“ in diesem Band. 


\section{RÄUMLICHE DIFFERENZIERUNG UND RAUMTYPEN}

Zum Zweck der Analyse bedarf es nicht nur in der Raum- und Stadtplanung sowie der Raumordnung, sondern ebenso in der Politik sowie der Wirtschaft einer Abgrenzung und Kategorisierung des Raumsystems. Seit dem frühen zwanzigsten Jahrhundert wurde für die Differenzierung von Raumtypen eine Vielzahl unterschiedlicher Methoden entwickelt, um auf Basis einheitlicher Kriterien räumliche Kategorien zu bilden. Jüngere Ansätze umfassen beispielsweise Raumtypen laut dem Bundesinstitut für Bau-, Stadt- und Raumforschung (BBSR 2010), Verkehrsraumtypen (Matthes/Gertz 2014) oder eine Urban-Rural-Typologie (Statistik Austria 2017). Die Verfahren unterscheiden sich zumeist in ihrer Berücksichtigung von (a) funktionalen Merkmalen, wie beispielsweise den Verkehrsströmen, Pendlerströmen und Versorgungsbeziehungen, sowie (b) Strukturmerkmalen, wie beispielsweise den Ausdehnungen des bebauten Gebietes, der Morphologie, der Bevölkerungs- und Arbeitsplatzdichte, der demographisch-soziologischen Bevölkerungsstruktur oder den Erwerbstätigenanteilen in Wirtschaftssektoren.

Während die Differenzierung von Siedlungsgebieten sich klassischerweise insbesondere mit der Frage nach der Abgrenzung von Stadt, Umland und ländlichem Raum befasst, betrachten andere Modelle die innere Gliederung des sogenannten Stadt-Land-Kontinuums (vgl. Borsdorf/Bender 2010: 250). Mit der Stadtregion lieferte insbesondere Boustedt (1953, 1970, 1975) im deutschsprachigen Raum eine wesentliche Grundlage für die Differenzierung von Raumeinheiten innerhalb von Stadtregionen. Das Modell basiert auf einer Kombination aus verschiedenen funktionalen Merkmalen (Verflechtungsgrad) und Strukturmerkmalen (Einwohnerzahl, Bevölkerungsdichte, Agrarquote) und diente als Basis für die spätere Konzeption der „Verdichtungsräume“, die zuletzt 1993 für Deutschland erneuert wurden (Borsdorf/Bender 2010).

Angesichts fortschreitender Zersiedelungstendenzen erweist sich der Gegensatz zwischen urbanen und ländlichen Gebieten allerdings als immer weniger produktiv. Zielführender scheint es, unterschiedliche Konstellationen des Urbanen oder unterschiedliche urbane Intensitäten zu differenzieren (Kretz/Kueng 2016). Zu den Ansätzen, die sich von einem traditionellen Verständnis der Stadt-Land-Dichotomie abwenden, zählen unter anderem die seit 1986 entwickelten „siedlungsstrukturellen Gebietstypen“ (Borsdorf/Bender 2010). Hierbei werden auf Basis der Einwohnerdichte sieben Regionstypen differenziert, die drei Grundtypen („Agglomerationsräume“, „Verstädterte Räume“ und „Ländliche Räume“) zugeordnet werden. Auch die vom BBSR entwickelten Raumtypen 2010 dienen einer flächendeckenden Typisierung des Raumes und sehen von einer Abgrenzung nach administrativen Grenzen ab. Ihre Klassifizierung beruht auf den drei Basisstrukturmerkmalen der Siedlungsstruktur (Bevölkerungsdichte und Siedlungsflächenanteil), Lage (Erreichbarkeit und Zentralität) und Wirtschaftskraft (BBSR 2010).

Im Rahmen der bisherigen Betrachtungen zu räumlichen Wirkungsweisen des automatisierten und vernetzten Fahrens wurde diese Art der räumlichen Differenzierung kaum berücksichtigt. Der Fokus lag bisher weitgehend auf einer makroskopischen Modellierung räumlicher und verkehrlicher Wirkungen des automatisierten und vernetzten Fahrens und dahingehend insbesondere auf städtischen Gebieten (Soteropoulos et al. 2019). 


\section{RÄUMLICHE WIRKUNGSWEISEN DES AUTOMATISIERTEN FAHRENS}

In den vergangenen Jahren wurde eine Reihe von Studien zu den möglichen räumlichen Wirkungen des automatisierten und vernetzten Fahrens veröffentlicht. Eine vielzitierte Grundlage liefert die Forschungsarbeit von Milakis et al. (2017), wonach die Wirkungsweisen des automatisierten Fahrens in primäre, sekundäre und tertiäre Effekte unterteilt werden können:

- Primäre Effekte: Verkehrsaufkommen (Straßenkapazität/Staubildung und Fahrleistung, i. e. gefahrene Fahrzeugkilometer), Zeitvorteile (Zeitwahrnehmung und Reisekomfort) und Verkehrsmittelwahl

- Sekundäre Effekte: Fahrzeugbesitz, Standortwahl und Flächennutzung, Verkehrsinfrastruktur

- Tertiäre Effekte: Energieverbrauch (Treibstoffeinsparungen), Umweltbelastung, Verkehrssicherheit, soziale Gerechtigkeit, Wirtschaft und Gesundheit

Speziell die verkehrlichen und räumlichen Wirkungen des automatisierten Fahrens wurden in zahlreichen Simulationsstudien untersucht (Soteropoulos et al. 2019). Dabei liegt der Fokus oftmals auf der szenarienbasierten Modellierung möglicher Veränderungen in der Verkehrsnachfrage, der Siedlungsstruktur und der Flächennutzung, die durch unterschiedliche Faktoren bedingt werden, wie den Wandel in der Verkehrsleistung, den Modal Split, den Parkplatzbedarf sowie die Standortwahl von Haushalten und Betrieben.

Während frühere Studien und Modellierungen meist von einem Zustand der vollständigen Durchdringung hochautomatisierter Fahrzeuge im SAE-Level 5 ausgehen (SAE 2018), wird er in späteren Studien vermehrt in Frage gestellt (Mitteregger et al. 2020, Soteropoulos et al. 2020). Stattdessen rückt eine längerfristige Phase des Übergangs in das Zentrum der Betrachtung, wonach unterschiedliche Automatisierungsgrade je nach Umfeldbedingungen zugelassen und in Mischverkehrsszenarien zum Einsatz kommen werden (siehe Beitrag 1 von Mitteregger und Banerjee in diesem Band). Die räumlichen Wirkungen des automatisierten und vernetzten Fahrens sind von der jeweiligen Angebotsart, der Siedlungsstruktur und dem Straßenraum sowie (geltenden) planungspolitischen Zielsetzungen abhängig. Auch gliedert sich die Entwicklung der Automatisierung in einen technologischen und organisatorischen Wandel der Mobilitätssysteme ein, durch den die Voraussetzungen für spätere Nutzungsweisen geschaffen werden. Dazu zählen beispielsweise das Car- und Ride-Sharing oder auch die Veränderungen im Logistik- und Warenverkehr (Beckmann/Brügger 2013, Gerdes/Heinemann 2019 sowie Beitrag 7 von Leerkamp et al. in diesem Band).

Die raumbezogenen Veränderungen, zu denen das automatisierte Fahren beitragen könnte, umfassen demnach den Wandel (1) des Parkens (z. B. straßengebundene Stellplätze, Parkplatzflächen, Parkgaragen), (2) der straßenräumlichen Flächenansprüche (z. B. Zu- und Ausstiegsbereiche etc.), (3) der erforderlichen Infrastruktur (z. B. digitale Vernetzung, Fahrbahnmarkierungen etc.) und (4) der Standortwahl von Betrieben und Haushalten. Da durch die genannten Veränderungen weiterführende Entwicklungen im Städtebau ebenso wie in der Siedlungsstruktur angestoßen werden können, lassen sich auch die räumlichen Folgen des automatisierten und vernetzten Fahrens in primäre und sekundäre Wirkungen einordnen (Abb. 1). 
Abbildung 1: Wirkungen des automatisierten Fahrens (aF) auf die Raumentwicklung

\section{Automatisiertes Fahren}

meint alle jene Angebotsformen, für die digital vernetzte und automatisierte Fahrzeuge zum Einsatz kommen, die ggf. elektrisch betrieben sind.

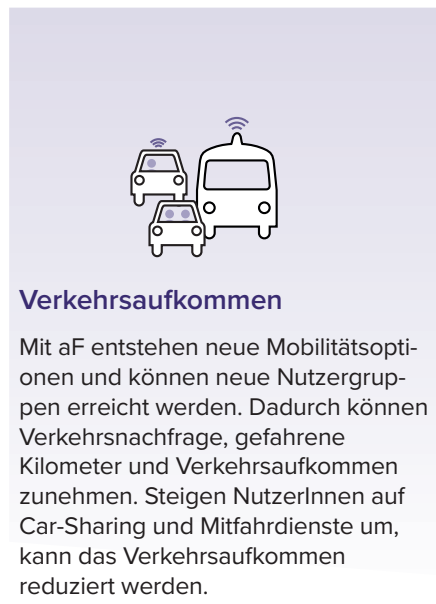

PRIMÄRE WIRKUNGEN

des automatisierten Fahrens

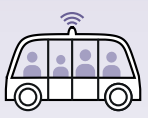

Zeitvorteile und Reisekomfort

Das aF kann aufgrund von Zeit- und Komfortvorteilen längere Pendlerfahrten begünstigen. Nutzerlnnen könnten die Zeit zu Unterhaltungsoder Arbeitszwecken nutzen.

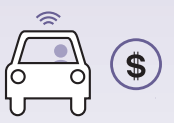

Nutzungs- und Betriebskosten

Kurzfristig liegen die Fixkosten eines Fahrzeugerwerbs höher als die eines konventionellen Fahrzeugs.

Allerdings können Effizienzvorteile die Reduktion der Nutzungs- und Betriebskosten begünstigen. Geringe Betriebskosten könnten öffentlichen Anbietern die Netzerweiterung erleichtern.

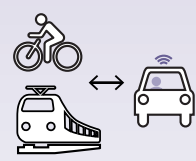

Verkehrsmittelwahl

Mit Einführung des aF kann es zu Veränderungen im Reiseverhalten kommen. NutzerInnen könnten anstelle des Fuß- oder Radweges vermehrt auf private Mitfahrdienste zurückgreifen. Auch könnte es zu einer Verlagerung weg vom öffentlichen Nahverkehr kommen.

\section{PRIMÄRE WIRKUNGEN auf den Siedlungsraum}

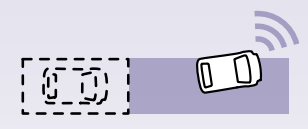

Veränderter Parkraumbedarf

Der Umstieg auf Mobilitätsdienste, Mikromobilität und das aF verändert Parkraumbedarf. Es besteht vermehrter Bedarf nach temporären Haltezonen statt nach straßengebundenen Parkplätzen. Dies hat

Auswirkungen auf die Verteilung des Straßenraums und die umliegende Bebauung.

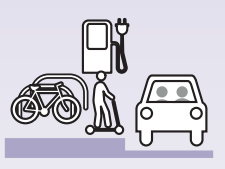

Flächenkonkurrenzen

Der Anstieg von Mobilitätsdiensten, Online-Handel und Mikromobilität erhöht den Druck auf die Nutzung und Flächenverteilung öffentlicher Räume. Setzt sich zudem das aF durch, steigt der Bedarf an Zu- und Ausstiegsflächen, deren Integration auf Mobilitätsmuster und räumliche Trenneffekte einwirkt.

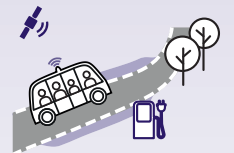

Ergänzende Infrastruktur

In den Einsatzgebieten des automatisierten Fahrens kann der Bedarf nach Infrastrukturanpassungen entstehen (z. B. digitale Infrastruktur, Zu- und Ausstiegsbuchten und Fahrbahnmarkierungen). Auch kann der Bedarf an E-Ladestationen und Servicemöglichkeiten steigen.

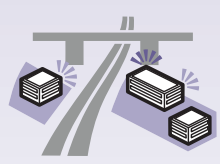

Verlagerung von Standorten

Kosten- und Komfortvorteile des aF könnten die Verlagerung von Standorten begünstigen. Dazu zählen: neue Standorte für dezentrale Sammelgaragen und Verteilerzentren des Warenverkehrs, die Abwanderung von Logistik- und Gewerbegebieten und die Wohnstandortwahl.

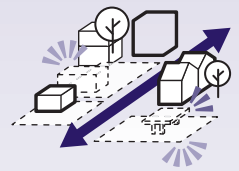

\section{Konversionsflächen}

Standortverlagerungen und ein Rückgang des Parkraumbedarfs setzen Entwicklungsflächen frei. Speziell Pkw-bezogene Flächen, wie Parkplätze, Garagen, Tankstellen etc. und Gewerbe- und Logistikgebiete. Räumliche Transformation und Nachverdichtung werden begünstigt.

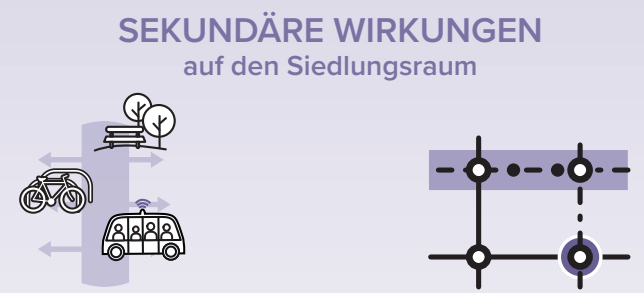

\section{Vielfalt im öffentlichen Raum}

Mit dem aF steigt die Vielfalt an Flächenansprüchen, die in Einklang zu bringen sind. Neben dem Rückbau von straßengebundenen Stellplätze und Fahrbahnbreiten ist die Priorisierung von Modi, Geschwindigkeiten und urbanen Qualitäten, z. B. räumliche Durchlässigkeit und Verweilräume, zu berücksichtigen.

\section{Neue Zentralitäten}

Wird das aF als Teil des ÖPNV-Netzes geplant, können an Mobilitätsknoten und neuen Haltepunkten Zentren entstehen. Auch kann die Transformation speziell suburbaner Gebiete eine Zentrenentwicklung fördern. Am Rande der Einsatzgebiete des aF können Flächenerfordernisse für Mobilitätshubs, Sammelund Verteilerzentren entstehen.

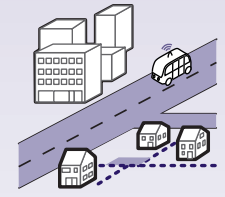

\section{Dynamik urbaner Dichte}

Aufgrund längerer Pendlerdistanzen kann die Zersiedelung und Abnahme der durchschnittlichen urbanen Dichte begünstigt werden. Auch könnten in Gebieten mit einem hohen Anteil an Konversionsflächen neue Dichten entstehen. Entlang der Einsatzrouten des aF könnte es zur Entwicklung räumlicher Korridore kommen. 


\subsection{PRIMÄRE RÄUMLICHE WIRKUNGEN}

Eine der viel genannten räumlichen Folgen ist beispielsweise der Rückgang des Parkraumbedarfs, der durch eine geringere Fahrzeuganzahl im Gesamtverkehr bedingt werden könnte. Allerdings steht speziell dieser Effekt in Abhängigkeit zu einer hohen Anzahl von Ride-SharingFahrten oder zumindest der flächendeckenden Durchsetzung von Car-Sharing (Zhang et al. 2015, Soteropoulos et al. 2019). Das Freiwerden von Parkplätzen ist demnach auf die künftige Akzeptanz der Mobility as a Service (MaaS) im Allgemeinen zurückzuführen. Würden die bereits heute verfügbaren geteilten Fahrtendienste nicht nur an Zuspruch gewinnen, sondern auch einen weitreichenden Verzicht privater Automobile zur Folge haben, so könnte dies die Rückgewinnung öffentlicher Räume begünstigen. Als konkrete Folge des automatisierten Fahrens wird allerdings die Standortverlagerung von Parkhäusern und Sammelgaragen angenommen. Würden in ausgewählten Stadträumen und Straßenzügen temporäre Zu- und Ausstiegsbereiche ausreichend bereitgestellt werden (siehe Beitrag 8 von Bruck et al. in diesem Band), so könnten Parkplätze reduziert und stattdessen in dezentralen Sammelgaragen gebündelt werden. Je nach Anbieterform und Flächenverfügbarkeit würden dazu unterschiedliche Standorte in Frage kommen (Lewis/Anderson 2020: 104).

Veränderungen im Straßenraum betreffen zudem die Flächenkonkurrenz zwischen unterschiedlichen Modi und Anbietern, die in Anbetracht steigender Lieferverkehre, plattformbasierter Fahrtendienste und von Angeboten der Mikromobilität bereits heute den Druck auf öffentliche Räume erhöhen (siehe Beitrag 8 von Bruck et al. sowie Beitrag 9 von Martin et al. in diesem Band). Auch könnte der automatisierte Verkehr räumliche Trennwirkungen begünstigen, da aufgrund einer Zunahme der gefahrenen Kilometer sowie einer Verstetigung des Verkehrsflusses die Querung von Straßenräumen für Zufußgehende und Radfahrende erschwert wird und an Durchlässigkeit verlieren könnte (Ghielmetti et al. 2017; siehe Beitrag 5 von Soteropoulos in diesem Band).

In beiderlei Hinsicht wird wesentlich sein, dass die Anforderungen für eine sichere Interaktion zwischen Verkehrsmodi mit jenen der räumlichen Verträglichkeit und Qualität in Einklang gebracht werden. Ergänzende Verkehrsinfrastrukturen umfassen sowohl jene der digitalen Infrastruktur (z. B. GPS, Satelliten, einheitliche Straßenkarten, „geo-fencing“ etc.) als auch baulich-materielle Infrastrukturvorkehrungen wie die Einrichtung von Zu- und Ausstiegsbereichen im öffentlichen Raum, an Mobilitätsknoten und Bahnhöfen (siehe Beitrag 8 von Bruck et al. in diesem Band). Im Sinne einer ressourcenschonenden Flächenentwicklung stellt insbesondere die Umnutzung bestehender Infrastrukturen (z. B. straßengebundene Stellplätze, Park-and-ride-Anlagen etc.) das Potential geringerer Flächenerfordernisse im Fall einer geteilten automatisierten Mobilität dar.

Letztlich wird davon ausgegangen, dass es in der Stadtregion zu einer Lageveränderung von Industrie- und Gewerbestandorten kommen könnte, die insbesondere entlang von höherrangigen und vom automatisierten und vernetzten Fahren frühzeitig erschlossenen Verkehrsachsen angesiedelt sind (Lewis/Anderson 2020, Mitteregger et al. 2020). Angestoßen durch heutige Entwicklungen im Onlinehandel ist es möglich, dass künftig kleinteilige und dezentrale Verteilerzentren in innerstädtischen Gebieten notwendig werden, währenddessen suburbane Logistikbetriebe weiter abwandern könnten. Zugleich könnten Vorteile des Reisekomforts, der Zeitersparnis und der Wirtschaftlichkeit dazu beitragen, dass längere Pendlerdistanzen in Kauf genommen werden und Arbeitsstätten ebenso wie Haushalte abwandern (Milakis et al. 2017, Litman 2020).

\subsection{SEKUNDÄRE RÄUMLICHE WIRKUNGEN}

Zu den sekundären Wirkungen des automatisierten und vernetzten Fahrens zählen in weiterer Folge u. a. freiwerdende Entwicklungs- und Konversionsflächen. So könnte die Automatisierung auf die Serviceorientierung im Mobilitäts- und Verkehrssystem („Shared Mobility“, Mobility as a 
Service) verstärkend wirken und dazu beitragen, dass nicht nur einzelne Parkplätze freiwerden, sondern der Flächenbedarf für sämtliche automobilbezogene Funktionen, insbesondere in Gewerbegebieten und Stadtrandlagen, in denen sie meist lokalisiert sind, zurückgehen könnte (siehe Beitrag 15 von Mitteregger und Soteropoulos in diesem Band). Speziell das Freiwerden dieser Flächenreserven bietet das Potential für räumliche Transformationen (Bruck 2019). Planungspolitische Maßnahmen, wie die Erneuerung von Stellplatzregulativen, die Umwidmung monofunktionaler Strukturen und der Fokus auf die Innenentwicklung, könnten in zuvor vernachlässigten Gebieten neue Entwicklungen anstoßen. Kommen diese in Abstimmung mit Einsatzgebieten des automatisierten und vernetzten Fahrens zur Anwendung, könnte speziell im Umfeld von Umstiegspunkten die räumliche Verdichtung begünstigt werden.

Automatisiertes und vernetztes Fahren verschärft darüber hinaus die Dringlichkeit, Vielfalt im öffentlichen Raum zu wahren und entsprechende Steuerungs- und Gestaltungsansätze zu setzen. Schon heute sind immer diversere Flächenansprüche (steigende Vielfalt an Verkehrsmitteln, Aufenthaltsbedarf, Erfordernisse der Klimaanpassung etc.) in Einklang zu bringen, weshalb davon auszugehen ist, dass mit der Einführung des automatisierten und vernetzten Fahrens der Nutzungsdruck im öffentlichen Raum weiter steigen wird. Ohne planungspolitische Maßnahmen würde das automatisierte und vernetzte Fahren zu einer erschwerten fußläufigen Durchlässigkeit von Straßenräumen beitragen. Würden sich allerdings hohe Besetzungsraten und das geteilte Fahren durchsetzen, besteht das Potential, straßengebundene Stellplätze rückzubauen und Fahrbahnbreiten zu schmälern sowie die Priorisierung von Modi, Geschwindigkeiten und Nutzungsformen grundsätzlich neu zu denken.

Werden Anwendungen des automatisierten und vernetzten Fahrens in diesem Sinne als Teil des öffentlichen Verkehrsnetzes geplant, könnte sich dies auf die Zentrenentwicklung und räumliche Konzentrationen auswirken. Bereits durchgeführte Studien zeigen dahingehend positive Wirkungstendenzen hinsichtlich lokaler Urbanisierungsprozesse und innerstädtischem Bevölkerungswachstum (Soteropoulos et al. 2019, WEF 2018).

Insbesondere an Haltepunkten, Mobilitätsknoten und neuen Mobilitätshubs ist mit einem Anstieg der Zentralitäten zu rechnen, die mittels entsprechender Planungsschwerpunkte zu fördern wären. Zudem kann auch die bereits genannte Umwidmung von Siedlungsstrukturen, die bislang monofunktional und durch automobilbezogene Funktionen geprägt waren, einen Wandlungsimpuls für neue Zentrenentwicklungen liefern. Langfristig kann eine Verlagerung räumlicher Dichten angenommen werden, die sich in Form von räumlichen Korridoren innerhalb der Einzugsbereiche des automatisierten und vernetzten Fahrens ausprägen könnte (Larco/ Tierney 2020: 123).

Die genannte Verlagerung könnte sich nicht zuletzt in Form einer Standortdynamik und Veränderung von Siedlungsdichten ausprägen - insbesondere, wenn automatisierte und vernetzte Fahrzeuge (avF) nicht als Teil des öffentlichen Verkehrs eingesetzt werden. Dominiert der Nutzen automatisierter Privatfahrzeuge, könnte die stadtregionale Zersiedelung begünstigt werden (Milakis et al. 2017, Litman 2020). Dahingehend ist anzunehmen, dass periphere Gebiete an Zuwachs gewinnen, währenddessen zentrale Gebiete aufgrund der Abwanderung bestimmter Nutzungen an Dichte verlieren könnten (Larco/Tierney 2020: 122).

Die Ungewissheit dieser Entwicklungspfade beruht nicht zuletzt auf der Erwartung, dass die kommenden Jahrzehnte von einer Gleichzeitigkeit unterschiedlicher Automatisierungsgrade und Anwendungsformen geprägt sein werden. Zudem lässt die räumliche Komplexität europäischer Straßenräume und die kostenintensive Aufrüstung vorhandener Infrastrukturnetze darauf schließen, dass die Einführung von avF auf ausgewählte Netzabschnitte und besonders geeignete Bereiche beschränkt sein wird (Mitteregger et al. 2020, Soteropoulos et al. 2020). 


\section{RÄUMLICHE DIFFERENZIERTE WIRKUNGSWEISEN DES AUTOMATISIERTEN FAHRENS}

\begin{abstract}
Besonders in Anbetracht einer langen Phase des Übergangs gilt es zu beachten, dass die genannten Wirkungsweisen nicht in allen Siedlungsstrukturen und Stadträumen und nicht im selben Ausmaß zum Tragen kommen werden. Ein Blick auf den heutigen Einsatz von „Transportation Network Companies" (TNCs) zeigt, dass die Technologie besonders in dichten, urbanen Zentren und unter wohlhabenden NutzerInnen zuerst Anklang fand. Dieser Aspekt ist insofern auch für das automatisierte Fahren lehrreich, als dass Ride-Sharing-Modelle in Gebieten hoher Dichte und auf kurzen Distanzen zwischen Quell- und Zielort am effizientesten zu betreiben sind. Je höher die Nutzerdichte, desto mehr Fahrten können mit weniger Fahrzeugen gebündelt werden (vgl. Larco/Tierney 2020). Andererseits werden die ersten Pilotprojekte selbstfahrender Minibusse und Fahrtendienste bevorzugt in suburbanen bis ländlichen Räumen getestet, da breite Straßennetze, niedrige Umweltkomplexität und geringe Verkehrsaufkommen technologische Erprobungen begünstigen. Auch stellt die Zubringerfunktion automatisierter Minibusse eine bevorzugte Angebotsform aus Sicht der städtischen Verkehrsbetriebe und Planungsämter dar.
\end{abstract}

Einzelne der bisher durchgeführten Studien zu automatisiertem Fahren betonen die räumliche Differenzierung der Wirkungsweisen des automatisierten Fahrens und den Punkt, dass diese unterschiedlich zum Ausdruck kommen werden. So beschäftigten sich beispielsweise Zhang und Wang (2020) sowie Kondor et al. (2020) mit den Wirkungen von avF-Sharing auf den Parkraumbedarf und die Flächennutzung. In den Studien konnte für die betrachteten Stadtregionen (Atlanta und Singapur) gezeigt werden, dass sich die Veränderungen ungleichmäßig vom Stadtkern bis zu den Vororten auswirken würden. Auch andere Studien (z. B. Legêne et al. 2020, Gelauff et al. 2017, Zhang 2017, Thakur et al. 2016) konnten ungleich verteilte Wirkungen von avF auf die Flächennutzung in Städten bzw. Stadtregionen aufzeigen.

Um Potentiale und Risiken räumlicher Entwicklungspfade zu konkretisieren, werden weitere Studien benötigt, die die räumlich differenzierten Wirkungsweisen des automatisierten Fahrens berücksichtigen und das Ausmaß potentieller Wirkungen in unterschiedlichen Stadtstrukturen und Gebieten aufzeigen. Damit sind nicht nur die oben genannten Veränderungen in der Flächennutzung oder des Parkraumbedarfs gemeint, sondern ebenso der potentielle Wandel im öffentlichen Raum oder Transformationen bestimmter Gebietstypen. Im Rahmen dieses Sammelbands widmen sich einzelne Beiträge dieser Aufgabe und behandeln einige der zuvor angeführten Wirkungsweisen vor dem Hintergrund unterschiedlicher Siedlungsstrukturen und Stadträume. Der Beitrag von Mitteregger und Soteropoulos (siehe Beitrag 15 in diesem Band) macht dies beispielsweise explizit für die Wirkungen von avF hinsichtlich freiwerdender Entwicklungs- und Konversionsflächen deutlich. Bruck et al. (siehe Beitrag 8 in diesem Band) differenzieren die Betrachtung räumlicher Schnittstellen im öffentlichen Raum je nach Siedlungsstrukturen, während Soteropoulos (siehe Beitrag 5) die Verträglichkeit und die räumlichen Trennwirkungen des automatisierten Verkehrs in unterschiedlichen Straßenräumen untersucht. 


\section{LITERATUR}

Angerer, F., und G. Hadler 2005. „Folgen und Wirkungen des Verkehrs - Städtebauliche Folgen“, in Stadtverkehrsplanung. Grundlagen, Methoden, Ziele 2, 152-159.

BBSR (Bundesinstitut für Bau-, Stadt- und Raumforschung) 2010. „Laufende Raumbeobachtung Raumabgrenzungen. Raumtypen 2010“. Bonn. https://tinyurl.com/yyp9cyea (28.12.2020).

Beckmann, J., und A. Brügger 2013. „Kollaborative Mobilität“, in Internationales Verkehrswesen 65, 57-59.

Bertolini, L. 2012. „Integrating Mobility and Urban Development Agendas - A Manifesto“, in disP - The Planning Review (48) 1, 16-26.

Bertolini, L. 2017. Planning the Mobile Metropolis: Transport for People, Places and the Planet. London: Palgrave/Red Grove Press.

Bormann, O. 2014. „Aktuelle Verkehrslage - Von der Rückgewinnung urbaner Infrastruktur“, in Architektur im Kontext, hg. v. K. von Keitz und S. Voggenreiter. Berlin: Jovis, 96-108.

Borsdorf, A., und O. Bender 2010. Allgemeine Siedlungsgeographie. Köln/Weimar/Wien: Böhlau.

Boustedt, O. 1953. „Die Stadtregion. Ein Beitrag zur Abgrenzung städtischer Agglomerationen“, in Allgemeines statistisches Archiv 37, 13-26.

Boustedt, O. 1970. „Stadtregionen“, in Handwörterbuch der Raumforschung und Raumordnung, hg. v. Akademie für Raumforschung und Landesplanung, 2. Auflage. Hannover: Gebrüder Jänecke, 3207-3237.

Boustedt, O.1975. „Grundriß der empirischen Regionalforschung. Teil1: Raumstrukturen“, Taschenbücher zur Raumplanung Band 4. Hannover: Hermann Schroedel.

Bruck, E. M. 2019. „Automatisierte Mobilitätsdienste als Wandlungsimpuls für suburbane Räume?“, in Broadacre city 2.0 - postfossil. Ein urbanistisches Szenario für 2050, hg. v. J. Fiedler. Graz: Haus der Architektur.

Gelauff, G., I. Ossokina und C. Teulings 2017. „Spatial effects of automated driving: dispersion, concentration or both?“, Working Paper, 18.9.2107. Download unter https://tinyurl.com/2bsd2ttn (28.12.2020) .

Gerdes, J., und G. Heinemann 2019. „Urbane Logistik der Zukunft - ganzheitlich, nachhaltig und effizient", in Handel mit Mehrwert: Digitaler Wandel in Märkten, Geschäftsmodellen und Geschäftssystemen, hg. v. G. Heinemann, H. M. Gehrckens und T. Täuber. Wiesbaden: Gabler, 397-420.

Ghielmetti, M., R. Steiner, J. Leitner, M. Hackenfort, S. Diener und H. Topp 2017. „Forschungsprojekt SVI 2011/023 Flächiges Queren in Ortszentren: Langfristige Wirkung und Zweckmässigkeit". Bern: Verkehrsteiner AG. https://tinyurl.com/y8ys7453 (28.12.2020).

Kasraian, D., K. Maat, D. Stead und B. van Wee 2016. „Long-term impacts of transport infrastructure networks on land-use change: An international review of empirical studies“, in Transport Reviews (36) 6, 772-792.

Kondor, D., P. Santi, D.-T. Le, X. Zhang, A. Millard-Ball und C. Ratti 2020. „Addressing the ,minimum parking problem for on-demand mobility“, in Scientific Reports 10, 15885. Download unter https:// tinyurl.com/3yuvj25a (28.12.2020).

Kretz, S., und L. Kueng (Hrsg.) 2016. Urbane Qualitäten - Ein Handbuch am Beispiel der Metropolitanregion Zürich. Zürich: Edition Hochparterre.

Larco, N., und G. Tierney 2020. „Impacts on Urban Design“, in Multilevel Impacts of Emerging Technologies on City Form and Development, hg. v. A. Howell und K. Lewis Chamberlain. Eugene, OR: University of Oregon, 115-141. https://tinyurl.com/ybdh7udt (28.12.2020).

Legêne, M. F., W. L. Auping, G. Correia und B. van Arem 2020. „Spatial impact of automated driving in urban areas“, in Journal of Simulation 14, 295-303. https://tinyurl.com/yaxqvh3y (28.12.2020).

Lewis, R., und M. Anderson 2020. „Impacts on Land Use“, in Multilevel Impacts of Emerging Technologies on City Form and Development, hg. v. A. Howell und K. Lewis Chamberlain. Eugene, OR: University of Oregon, 97-113. https://tinyurl.com/ybdh7udt (28.12.2020).

Litman, T. 2020. „Autonomous Vehicle Implementation Predictions: Implications for Transport Planning“. Victoria, BC: Victoria Transport Policy Institute. https://www.vtpi.org/avip.pdf (4.6.2020).

Matthes, G., und C. Gertz 2014. „Raumtypen für Fragestellungen der handlungstheoretisch orientierten Personenverkehrsforschung“, ECTL Working Paper 45. Hamburg: TU Hamburg, Institut für Verkehrsplanung und Logistik. 
Milakis, D., B. van Arem und B. van Wee 2017. „Policy and society related implications of automated driving: A review of literature and directions for future research", in Journal of Intelligent Transportation Systems (21) 4, 324-348.

Mitteregger, M., E. M. Bruck, A. Soteropoulos, A. Stickler, M. Berger, J. S. Dangschat, R. Scheuvens und I. Banerjee 2020. AVENUE21. Automatisierter und vernetzter Verkehr: Entwicklungen des urbanen Europa. Berlin: Springer Vieweg.

SAE International 2018. „Taxonomy and Definitions for Terms Related to Driving Automation Systems for On-Road Motor Vehicles - J3016“, 15.6.2018. whttps://tinyurl.com/a5f53jus (20.4.2020).

Soteropoulos, A., M. Berger und F. Ciari 2019. „Impacts of automated vehicles on travel behaviour and land use: An international review of modelling studies“, in Transport Reviews (39) 1, 29-49.

Soteropoulos, A., M. Mitteregger, M. Berger und J. Zwirchmayr 2020. „Automated drivability: Toward an assessment of the spatial deployment of level 4 automated vehicles", in Transportation Research Part A: Policy and Practice 136, 64-84.

Statistik Austria 2017. „Urban-Rural-Typologie“. https://tinyurl.com/6y3yjkrc (4.6.2020).

Steckler, B. 2019. „Navigating New Mobility: Policy Approaches for Cities“, Urbanism Next Reports, October 2019. Eugene, OR: Urbanism Next Center, University of Oregon. Download unter https:// scholarsbank.uoregon.edu/xmlui/handle/1794/25190 (1.12.2020).

Thakur, P., R. Kinghorn und R. Grace 2016. „Urban form and function in the autonomous era“, Australasian Transport Research Forum 2016 Proceedings. Melbourne. https://tinyurl.com/yblrqyu4 (28.12.2020).

VCÖ (Verkehrsclub Österreich) 2019. „In Gemeinden und Regionen Mobilitätswende voranbringen“, VCÖ Mobilität mit Zukunft. https://tinyurl.com/b2pcxdh5 (4.6.2020).

WEF (World Economic Forum) 2018. „Reshaping Urban Mobility with Autonomous Vehicles: Lessons from the City of Boston“. https://tinyurl.com/2w6um58r (4.6.2020).

Wegener, M., und F. Fürst 1999. „Land-Use Transport Interaction - State of the Art“, Berichte aus dem Institut für Raumplanung 46. Dortmund: TU Dortmund.

Weidmann, U., R. Dorbritz, H. Orth, M. Scherer und P. Spacek 2011. „Einsatzbereiche verschiedener Verkehrsmittel in Agglomerationen. Schweizerische Eidgenossenschaft: Eidgenössisches Departement für Umwelt, Verkehr, Energie und Kommunikation UVEK. https://tinyurl.com/uncz38rk (4.6.2020).

Zhang, W. 2017. „The interaction between land use and transportation in the era of shared autonomous vehicles: A simulation model“, Dissertation, Georgia Institute of Technology, Atlanta, GA.

Zhang, W., und S. Guhathakurta 2018. „Residential Location Choice in the Era of Shared Autonomous Vehicles“, in Journal of Planning Education and Research, 1-14.

Zhang, W., S. Guhathakurta, J. Fang und G. Zhang 2015. „Exploring the impact of shared autonomous vehicles on urban parking demand: An agent-based simulation approach“, in Sustainable Cities and Society 19, 34-45.

Zhang, W., und K. Wang 2020. „Parking futures: Shared automated vehicles and parking demand reduction trajectories in Atlanta“, in Land Use Policy 91, 103963. https://tinyurl.com/4h6er3dc.

Open Access Dieser Beitrag wird unter der Creative Commons Namensnennung 4.0 International Lizenz (http://creativecommons.org/licenses/by/4.0/deed.de) veröffentlicht, welche die Nutzung, Vervielfältigung, Bearbeitung, Verbreitung und Wiedergabe in jeglichem Medium und Format erlaubt, sofern Sie den/die ursprünglichen Autor(en) und die Quelle ordnungsgemäß nennen, einen Link zur Creative Commons Lizenz beifügen und angeben, ob Änderungen vorgenommen wurden.

Die in diesem Beitrag enthaltenen Bilder und sonstiges Drittmaterial unterliegen ebenfalls der genannten Creative Commons Lizenz, sofern sich aus der Abbildungslegende nichts anderes ergibt. Sofern das betreffende Material nicht unter der genannten Creative Commons Lizenz steht und die betreffende Handlung nicht nach gesetzlichen Vorschriften erlaubt ist, ist für die oben aufgeführten Weiterverwendungen des Materials die Einwilligung des jeweiligen Rechteinhabers einzuholen.

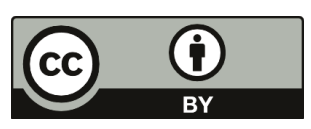

